\title{
MACRONUTRIENTES NA PRODUÇÃO DE MUDAS DE CANAFÍSTULA EM ARGISSOLO VERMELHO AMARELO DA REGIÃO DA ZONA DA MATA, MG
}

\author{
MACRONUTRIENTS IN PRODUCTION OF Peltophorum dubium (Spreng.) Taub. \\ SEEDLINGS IN RED YELLOW ULTISOL OF THE ZONA DA MATA, MG REGION
}

\author{
Cezar Augusto Fonseca e Cruz ${ }^{1}$ Haroldo Nogueira de Paiva ${ }^{2}$ \\ Ana Catarina Monteiro Carvalho Mori da Cunha ${ }^{3}$ Júlio César Lima Neves ${ }^{4}$
}

\begin{abstract}
RESUMO
O objetivo deste trabalho foi avaliar o efeito de doses de macronutrientes sobre o crescimento e os índices de qualidade de mudas de canafístula e determinar a dose recomendada dos mesmos para estabelecer um adequado programa de adubação. No trabalho, conduzido em casa de vegetação, utilizou-se amostras de Argissolo Vermelho Amarelo, retirado da camada abaixo de $20 \mathrm{~cm}$ de profundidade, como substrato. Delimitou-se o experimento por meio de matriz baconiana, avaliando-se três doses dos seis macronutrientes (nitrogênio, fósforo, potássio, cálcio, magnésio e enxofre) e dois tratamentos adicionais, um com doses de referência e outro sem adição de nutrientes. Adotou-se o delineamento inteiramente casualizado com quatro repetições. Foram analisados os parâmetros morfológicos e os índices de qualidade de mudas. Verificouse, através de análise dos contrastes ortogonais, resposta significativa de todas as variáveis estudadas à aplicação dos macronutrientes, exceto para a relação altura diâmetro (H/D) nos tratamentos com P, Ca e S. Não foi verificada resposta à aplicação de Ca para a maioria das características avaliadas, com exceção das relações altura de parte aérea por peso de matéria seca de parte aérea (H/MSPA) e peso de matéria seca de

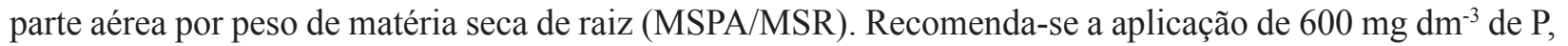
$150 \mathrm{mg} \mathrm{dm}^{-3}$ de K, 0,50 $\mathrm{cmol}_{\mathrm{c}} \mathrm{dm}^{-3} \mathrm{de} \mathrm{Mg}$ e $50 \mathrm{mg} \mathrm{dm}^{-3}$ de $\mathrm{S}$, e sugerem-se novos estudos com os nutrientes $\mathrm{N}$ e $\mathrm{Ca}$, para a produção de mudas de canafístula, usando a camada abaixo de $20 \mathrm{~cm}$ de profundidade de Argissolo Vermelho Amarelo como substrato.
\end{abstract}

Palavras-chave: nutrição de planta; reflorestamento; Peltophorum dubium.

\begin{abstract}
The objective of this work was to evaluate the effect of macronutrients doses upon the growth and quality indices of Peltophorum dubium seedlings and to determine the recommended doses for them to establish a suitable fertilization program. In the paper, carried out in a greenhouse, samples of 'Red Yellow Ultisol' were used, removed from the layer below, $20 \mathrm{~cm}$ deep, as a substratum. The experiment was designed in a Baconian Matrix, and three doses of the six macronutrients (nitrogen, phosphorus, potassium, calcium, magnesium and sulfur) and two additional treatments were evaluated, one with reference doses and the other one without the addition of nutrients. The entirely casual design was adopted, with four repetitions. The morphologic parameters and quality indices of seedlings were analyzed. Through the orthogonal contrasts, a significant response of all the variables studied was verified related to the macronutrient applied, except for the relation height/diameter (H/D) in the treatments with $\mathrm{Ca}, \mathrm{P}$ and $\mathrm{S}$. It was not verified response to $\mathrm{Ca}$ application for most characteristics evaluated, unless for the relation of the aerial part height per aerial
\end{abstract}

1. Engenheiro Florestal, MSc., Analista do Instituto Estadual de Florestas, Av. Doutor David Benedito Ottoni, 257, Jardim dos Estados, CEP 37701-069, Poços de Caldas (MG). ceaufoc1@yahoo.com.br

2. Engenheiro Florestal, Dr., Professor Associado do Departamento de Engenharia Florestal, Universidade Federal de Viçosa, Centro de Ciências Agrárias, CEP 36570-000, Viçosa (MG). hnpaiva@ufv.br

3. Engenheira Florestal, DS, Professora do Instituto Federal de Educação, Ciência e Tecnologia do Sudeste de Minas Gerais, Av. Dr. José Sebastião da Paixão, S/N, Lindo Vale, 36180-000, Rio Pomba, MG, Brasil, catarina_mori@yahoo.com.br

4. Engenheiro Agrônomo, Dr., Professor Adjunto do Departamento de Solos e Nutrição de Plantas, Universidade Federal de Viçosa, Centro de Ciências Agrárias, CEP 36570-000, Viçosa (MG). julio_n2003@yahoo.com.br

Recebido para publicação em 5/10/2009 e aceito em 26/08/2010 
part dry weight (H/MSPA) and aerial part dry weight per root dry weight (MSPA/MSR). The recommended doses were of $600 \mathrm{mg} \mathrm{dm}^{-3}$ of P, $150 \mathrm{mg} \mathrm{dm}^{-3}$ of K, $0,50 \mathrm{cmol}_{\mathrm{c}} \mathrm{dm}^{-3}$ of $\mathrm{Mg}$ and $50 \mathrm{mg} \mathrm{dm}^{-3}$ of S. Based on this work, further studies are suggested with $\mathrm{N}$ and $\mathrm{Ca}$, for seedlings production of the studied species using from the layer below $20 \mathrm{~cm}$ deep of the 'Red Yellow Ultisol' as the substratum.

Keywords: plant nutrition; reforestation; Peltophorum dubium.

\section{INTRODUÇÃO}

A canafístula (Peltophorum dubium (Spreng.) Taub.) é uma leguminosa arbórea nativa, de ampla dispersão geográfica; desempenha papel pioneiro em áreas abertas, capoeiras e matas degradadas (MACHIORI, 1997; CARVALHO, 2003). Carvalho (2003) classifica a espécie como pertencente, no estudo de sucessão, ao grupo das secundárias iniciais. A canafístula é medianamente tolerante ao frio, pode ser usada para a arborização urbana e cercas divisórias de diversas propriedades (VENTURIN et al., 1999) e, devido ao rápido crescimento e rusticidade, é ótima para a composição de reflorestamentos mistos de áreas degradadas de preservação permanente (LORENZI, 1992). Deve-se ressaltar ainda que apesar da leguminosa em estudo tratar-se de espécie não nodulífera (BARBERI et al., 1998; DIAS et al., 2007; COELHO et al., 2007), Dias et al. (2007) afirmam que as gramíneas se beneficiam do nitrogênio fixado pelas leguminosas, seja pela excreção direta de compostos nitrogenados pelas raízes, seja pela decomposição dos nódulos das raízes, da liteira e da serapilheira das mesmas, justificando a necessidade de se conhecer o comportamento da canafístula em relação às doses nitrogenadas.

A degradação de pastagens é um problema comum em diversas propriedades rurais de Minas Gerais, sendo causada pelo manejo inadequado das mesmas (CARVALHO, 1997). Assim sendo, as leguminosas arbóreas merecem destaque quando o assunto é a recuperação dessas áreas, isto porque essas espécies são pioneiras e agressivas, com elevada produção de biomassa e ocorrência em diferentes condições climáticas (BALIERO et al., 2001).

As leguminosas arbóreas possuem sistema radicular profundo, o qual propicia uma melhor infiltração de água no solo e controle da erosão, melhorando também a fertilidade do solo. Entretanto, uma das principais justificativas do uso de leguminosas em programas de recuperação de áreas degradadas é a sua capacidade de associação com bactérias fixadoras de nitrogênio, além de formar associação com micorrizas. No entanto, segundo Fernández et al. (1996), para a maioria dessas espécies são desconhecidas as exigências nutricionais, o que tem conduzido à adoção de adubações padronizadas, desconsiderando as exigências de cada espécie, impossibilitando assim a obtenção do potencial máximo de crescimento e a otimização dos insumos.

Especial atenção deve ser dada à produção de mudas de espécies florestais nativas, tendo em vista que, de acordo com Gonçalves et al. (2000), a produção das mesmas com qualidade e na quantidade desejada é uma das fases mais importantes para o estabelecimento de bons povoamentos florestais. Neste contexto, o conhecimento das exigências nutricionais das espécies arbóreas é de grande importância na recomendação adequada de fertilizantes na fase de viveiro, uma vez que proporcionará mudas de boa qualidade, refletindo maior potencial de sobrevivência e crescimento após o plantio, e como consequência, queda nas taxas de replantio e tratos culturais, o que se traduz em redução de custos (CARNEIRO, 1995). Além disso, algumas práticas como o uso de sementes com qualidade superior, juntamente com técnicas de implantação adequadas também são importantes para o sucesso dos programas de reflorestamento.

Apesar da grande potencialidade dos plantios com canafístula, pouco se conhece sobre suas exigências nutricionais e sobre as limitações ao seu crescimento, tanto em viveiro (na fase de muda), quanto em campo (em programas de plantio). Dentre as principais limitações podem-se destacar a baixa fertilidade natural da maioria dos solos do Brasil, com ênfase para os macronutrientes, tanto em culturas agrícolas quanto florestais, sendo esses nutrientes os exigidos em maior quantidade pelas plantas. No entanto, segundo Raij (1991) insuficiente atenção é dispensada nos trabalhos de pesquisa, sendo o conhecimento caracterizado por lacunas sobre aspectos considerados básicos.

A necessidade de adubação decorre do fato de que nem sempre o solo é capaz de fornecer todos os nutrientes que as plantas precisam para um adequado crescimento. Ainda, as características e quantidades de adubos a serem aplicados dependerão das necessidades nutricionais da espécie utilizada, da fertilidade do solo, da forma de reação dos adubos com o solo, da eficiência dos adubos e de fatores de ordem econômica.

Em razão da potencialidade da canafístula e devido à carência de informações sobre a exigência nutricional da espécie, os objetivos deste trabalho

Ci. Fl., v. 21, n. 3, jul.-set., 2011 
foram: avaliar o efeito da aplicação de doses de macronutrientes sobre o crescimento e qualidade das mudas de canafístula cultivada em Argissolo Vermelho Amarelo e; determinar a dose recomendada dos mesmos para a produção de mudas desta espécie.

\section{MATERIAIS E MÉTODOS}

O estudo foi conduzido em casa de vegetação no viveiro de pesquisa do Departamento de Engenharia Florestal da Universidade Federal de Viçosa, Viçosa, MG, no período de janeiro a junho de 2006.

Situado nas coordenadas $20^{\circ} 45^{\prime} \mathrm{S}$ e $42^{\circ} 55^{\prime} \mathrm{W}$ na Zona da Mata de Minas Gerais, o município de Viçosa possui clima classificado como tropical de altitude, com verões chuvosos e invernos frios e secos, do tipo Cwb, pelo sistema de Köeppen e possui precipitação média anual de $1.221 \mathrm{~mm}$.

Para a realização do estudo foram usadas amostras de um Argissolo Vermelho Amarelo (Argila $39 \%$; Silte $16 \%$; Areia grossa $27 \%$; Areia fina $18 \%$ ) retiradas da camada abaixo de $20 \mathrm{~cm}$ de profundidade, secas ao ar, e peneiradas em malha de $4 \mathrm{~mm}$ de diâmetro para então serem devidamente caracterizadas quimicamente (Tabela 1).

As amostras de solo utilizadas neste estudo foram provenientes de áreas próximas à Viçosa, sendo que o mesmo tem ocorrência predominante na região da Zona da Mata mineira, motivo pelo qual foi escolhido para a realização do experimento.

Os tratamentos foram obtidos segundo matriz baconiana (Turrent, 1979), na qual um dos nutrientes é fornecido em quantidades variáveis, enquanto os outros são mantidos em um nível referencial. Com esta matriz pretendeu-se avaliar seis nutrientes (nitrogênio, fósforo, potássio, cálcio, magnésio e enxofre) em três diferentes doses e, ainda dois tratamentos adicionais, sendo um com doses de referência e outro sem adição de nutrientes, totalizando 20 tratamentos, que foram dispostos em delineamento inteiramente casualizado, com quatro repetições. Os tratamentos foram delimitados de maneira que, quando a quantidade de um nutriente estivesse variando, as doses dos demais estariam fixas, sendo que a variação das doses e nutrientes em cada tratamento podem ser visualizadas na Tabela 2.

TABELA 1: Análise química das amostras de solo utilizadas na produção das mudas antes da aplicação dos tratamentos.

TABLE 1: Chemical analysis of the soil samples used for the production of the seedlings before the treatments.

\begin{tabular}{|c|c|c|c|c|c|c|c|c|c|}
\hline Solo & $\begin{array}{l}\mathrm{pH} \\
\mathrm{H}_{2} \mathrm{O}\end{array}$ & $\begin{array}{l}\mathrm{P} \quad \mathrm{K} \\
\mathrm{mg} \mathrm{dm}{ }^{-3}\end{array}$ & $\mathrm{Ca}^{2+}$ & $\mathrm{Mg}^{2+}$ & $\begin{array}{l}\mathrm{Al}^{3+} \\
\mathrm{cmol}_{\mathrm{c}} \mathrm{dm}^{-3}\end{array}$ & SB & (T) & $\mathrm{V}_{\%}^{\mathrm{m}}$ & $\begin{array}{c}\text { MO } \\
\text { dag kg-1 }\end{array}$ \\
\hline $\begin{array}{c}\text { Argissolo Vermelho } \\
\text { Amarelo }\end{array}$ & 6,00 & 2,10 & 1,60 & 0,10 & 3,63 & 1,74 & 5,37 & 32 & 2,82 \\
\hline
\end{tabular}

Em que: $\mathrm{pH}$ em água, $\mathrm{KCl}$ e $\mathrm{CaCl}_{2}^{-}$- Relação 1:2,5; $\mathrm{SB}$ = Soma de bases trocáveis; $\mathrm{P} \mathrm{e} \mathrm{K}$ - Extrator Mehlich 1; $\mathrm{V}=$ Índice de Saturação por bases; CTC $(\mathrm{T})=$ Capacidade de troca catiônica (pH 7,0); MO - C. Org x 1,724 - Método Walkley-Black; $\mathrm{Ca}^{2+}, \mathrm{Mg}^{2+} \mathrm{e} \mathrm{Al}^{3+}$ - Extrator $\mathrm{KCl} 1 \mathrm{~mol} \mathrm{~L}^{-1} ; \mathrm{m}=$ Saturação por alumínio; $\mathrm{H}+\mathrm{Al}$ - Extrator Acetato de Cálcio $0,5 \mathrm{~mol} \mathrm{~L}^{-1}-\mathrm{pH} 7,0$.

TABELA 2: Tratamentos, obtidos pela matriz baconiana, com as doses dos nutrientes aplicadas no substrato utilizado para a produção das mudas.

TABLE 2: Treatments obtained through the Baconian Matrix, with the doses of nutrients applied in the substratum used for the production of the seedlings.

\begin{tabular}{cccc}
\hline TRATAMENTOS & UNIDADES & TRATAMENTOS & UNIDADES \\
\hline DR & $\mathrm{mg} / \mathrm{dm}^{3} / \mathrm{cmol}_{\mathrm{c}} / \mathrm{dm}^{3}$ & $\mathrm{~K}=200$ & $\mathrm{mg} \mathrm{dm}^{-3}$ \\
Solo sem correção & - & $\mathrm{Ca}=0,8$ & $\mathrm{cmol}_{\mathrm{c}} \mathrm{dm}^{-3}$ \\
$\mathrm{~N}=50$ & $\mathrm{mg} \mathrm{dm}-3$ & $\mathrm{Ca}=1,2$ & $\mathrm{cmol}_{\mathrm{c}} \mathrm{dm}^{-3}$ \\
$\mathrm{~N}=150$ & $\mathrm{mg} \mathrm{dm}$ & $\mathrm{Ca}=1,4$ & $\mathrm{cmol}_{\mathrm{c}} \mathrm{dm}^{-3}$ \\
$\mathrm{~N}=200$ & $\mathrm{mg} \mathrm{dm}$ & $\mathrm{Mg}=0,2$ & $\mathrm{cmol}_{\mathrm{c}}^{-3} \mathrm{dm}^{-3}$ \\
$\mathrm{P}=150$ & $\mathrm{mg} \mathrm{dm}$ & $\mathrm{Mg}=0,6$ & $\mathrm{cmol}_{\mathrm{c}}^{-3} \mathrm{dm}^{-3}$ \\
$\mathrm{P}=450$ & $\mathrm{mg} \mathrm{dm}$ & $\mathrm{Mg}=0,8$ & $\mathrm{cmol}_{\mathrm{c}}^{-3} \mathrm{dm}^{-3}$ \\
$\mathrm{P}=600$ & $\mathrm{mg} \mathrm{dm}$ & $\mathrm{S}=20$ & $\mathrm{mg} \mathrm{dm}^{-3}$ \\
$\mathrm{~K}=50$ & $\mathrm{mg} \mathrm{dm}^{-3}$ & $\mathrm{~S}=60$ & $\mathrm{mg} \mathrm{dm}^{-3}$ \\
$\mathrm{~K}=150$ & $\mathrm{mg} \mathrm{dm}$ & $\mathrm{S}=80$ & $\mathrm{mg} \mathrm{dm}^{-3}$ \\
\hline
\end{tabular}

Em que: $\mathrm{DR}=$ Valores da dose de referência: $\mathrm{N}=100 \mathrm{mg} \mathrm{dm}^{-3} ; \mathrm{P}=300 \mathrm{mg} \mathrm{dm}^{-3} ; \mathrm{K}=100 \mathrm{mg} \mathrm{dm}^{-3} ; \mathrm{Ca}=1 \mathrm{cmol}_{\mathrm{c}} \mathrm{dm}^{-3}$; $\mathrm{Mg}=0,4 \mathrm{cmol}_{\mathrm{c}} \mathrm{dm}^{-3} ; \mathrm{S}=40 \mathrm{mg} \mathrm{dm}^{-3}$. 
A unidade experimental foi constituída por um vaso de polietileno rígido com capacidade de $2,1 \mathrm{dm}^{3}$ de solo e uma muda. A espécie selecionada para realização deste trabalho foi a canafístula, cujas sementes foram originárias do Laboratório de Análise de Sementes (LASO) da Fundação Estadual de Pesquisa Agropecuária (FEPAGRO) do Rio Grande do Sul.

Foi pesado $2,1 \mathrm{~kg}$ do solo usado como substrato na produção de mudas e, em seguida, acondicionado em sacos plásticos. Posteriormente, foi adicionado ao solo doses de corretivos, sendo empregada um mistura de $\mathrm{CaCO}_{3}$ e $\mathrm{MgCO}_{3}$ nas quantidades definidas pelos tratamentos (Tabela 2) e homogeneizados. Depois da aplicação dos corretivos seguiu-se um período de incubação de 30 dias, mantendo o teor de água próximo à capacidade de campo.

Decorridos os 30 dias, foram adicionados os demais nutrientes, de acordo com as quantidades definidas na Tabela 2. A aplicação de nitrogênio e potássio foi parcelada em $0,30,50,70$ e 90 dias após a semeadura.

Antes da semeadura aplicou-se uma solução de micronutrientes, exceto no tratamento sem correção do solo, composta por: boro (B) $(0,81$ $\mathrm{mg} / \mathrm{dm}^{3}$ de $\left.\mathrm{H}_{3} \mathrm{BO}_{3}\right)$, cobre $(\mathrm{Cu})\left(1,33 \mathrm{mg} / \mathrm{dm}^{3}\right.$ de $\left.\mathrm{CuSO}_{4} \cdot 5 \mathrm{H}_{2} \mathrm{O}\right)$, molibdênio (Mo) $\left(0,15 \mathrm{mg} / \mathrm{dm}^{3} \mathrm{de}\right.$ $\left.\left(\mathrm{NH}_{4}\right)_{6} \mathrm{Mo}_{7} \mathrm{O}_{24} \cdot 4 \mathrm{H}_{2} \mathrm{O}\right)$, manganês $(\mathrm{Mn})(3,66 \mathrm{mg} /$ $\mathrm{dm}^{3}$ de $\mathrm{MnCl}_{2} \cdot \mathrm{H}_{2} \mathrm{O}$ e zinco $(\mathrm{Zn})\left(4,0 \mathrm{mg} / \mathrm{dm}^{3}\right.$ de $\mathrm{ZnSO}_{4} \cdot 7 \mathrm{H}_{2} \mathrm{O}$ ) como indicado por Alvarez V. (1974).

Fez-se a semeadura de 10 sementes diretamente nos vasos. O primeiro raleio foi realizado 15 dias após a emergência das plântulas deixando-se duas plântulas por vaso, e o segundo aos 30 dias, permanecendo então apenas uma plântula por vaso. O teor de umidade do solo foi controlado através de monitoramento diário a fim de mantê-lo próximo a $60 \%$ da capacidade de campo.

Aos 120 dias após a semeadura, realizouse a medição da altura $(\mathrm{H})$ utilizando-se régua graduada em centímetros e, diâmetro do coleto (DC) das mudas com paquímetro com precisão de $0,01 \mathrm{~mm}$, quando então se deu por encerrado o experimento. Em seguida, as plantas foram colhidas e subdivididas em raiz e parte aérea, lavadas em água destilada e postas a secar em estufa com circulação forçada de ar a $60^{\circ} \mathrm{C}$ até que atingissem peso constante.

A determinação do peso de matéria seca de raiz (MSR) e peso de matéria seca da parte aérea (MSPA) foi realizada em balança analítica com precisão de $0,01 \mathrm{~g}$, e o peso de matéria seca total (MST) foi obtido pela soma da MSR e da MSPA. Com esses dados, calculou-se os índices de qualidade de mudas: altura de parte aérea por diâmetro do coleto (H/D), altura de parte aérea por peso de matéria seca de parte aérea (H/MSPA), peso de matéria seca de parte aérea por peso de matéria seca de raiz (MSPA/MSR), bem como o Índice de Qualidade de Dickson (IQD), de acordo com a fórmula (DICKSON et al., 1960):

$$
\mathrm{IQD}=\mathrm{MST} /(\mathrm{H} / \mathrm{D}+\mathrm{MSPA} / \mathrm{MSR})
$$

Onde: MST - Peso de matéria seca total (g); H - Altura de parte aérea (cm); D - Diâmetro do coleto $(\mathrm{mm})$; MSR - Peso de matéria seca de raiz $(\mathrm{g})$ e; MSPA - Peso de matéria seca da parte aérea (g).

Os dados foram interpretados estatisticamente por meio de contrastes ortogonais, análises de variância e regressão, utilizando-se o programa SAEG (Sistema de Análises Estatística e Genética) (EUCLYDES, 1997) e o Statistica. A partir das análises de variância individuais estimouse o erro experimental e de regressão e procedeu-se a escolha do modelo com melhor ajuste aos dados, com base no coeficiente de determinação $\left(\mathrm{R}^{2}\right)$. $\mathrm{O}$ ajuste das equações de regressão foi feito testandose os respectivos coeficientes pelo teste "t", de Student, com base no quadrado médio do resíduo da ANOVA em nível de 1, 5 e 10\% de probabilidade. Diante de dois ou mais modelos com coeficientes significativos, optou-se por aquele que apresentou maior $\mathrm{R}^{2}$ e, com essas equações, determinaram-se as doses recomendadas dos macronutrientes, para obtenção de $90 \%$ dos valores máximos estimados das variáveis estudadas.

\section{RESULTADOS E DISCUSSÃO}

Os contrastes ortogonais entre os tratamentos com e sem fertilizantes (dose zero) indicaram que os tratamentos com adição de macronutrientes foram significativos, exceto para a relação $\mathrm{H} / \mathrm{D}$ nos tratamentos com $\mathrm{P}, \mathrm{Ca}$ e $\mathrm{S}$. Isto indica que a fertilização com macronutrientes possibilitou significativo aumento do crescimento e qualidade das mudas de canafístula.

As médias de crescimento e qualidade de mudas (Tabela 3 ) observadas em todas as variáveis analisadas, exceto para as características H/D e H/ MSPA, no tratamento sem fertilização, indicam que os teores de nutrientes originalmente existentes no

Ci. Fl., v. 21, n. 3, jul.-set., 2011 
solo estudado, à exceção do Ca, são baixos. Verificase, ainda, que os valores médios nas diversas características, em geral, aumentaram em função da aplicação de doses crescentes de macronutrientes, à exceção da relação H/MSPA. Ainda no que tange às médias observadas para os índices de qualidade de mudas H/D e H/MSPA a literatura pertinente é unânime em afirmar que quanto menor o valor destes índices de qualidade de mudas, maior será a qualidade das mudas produzidas. Diante disto, nota-se que as mudas testemunhas apresentavam qualidade inferior às demais. Essa resposta era esperada, pois os nutrientes desempenham diversas funções na planta, logo, sua omissão irá comprometer diversos processos metabólicos, o que implica em queda no ritmo de crescimento e desenvolvimento das plantas, bem como pode ser prejudicial à qualidade das mudas produzidas.

TABELA 3: Crescimento de mudas de canafístula em razão da aplicação de N, P, K, Ca, Mg e S para as seguintes características: altura (H), diâmetro do coleto (DC), peso de matéria seca da parte aérea (MSPA), peso de matéria seca de raiz (MSR), peso de matéria seca total (MST), altura de parte aérea por diâmetro do coleto (H/D), altura de parte aérea por peso de matéria seca de parte aérea (H/MSPA), peso de matéria seca de parte aérea por peso de matéria seca de raiz (MSPA/MSR) e Índice de Qualidade de Dickson (IQD), aos 120 dias após a semeadura, cultivadas em Argissolo Vermelho Amarelo.

TABLE 3: Growth of Peltophorum dubium seedlings due to $\mathrm{N}, \mathrm{P}, \mathrm{K}, \mathrm{Ca}, \mathrm{Mg}$ and S application for the following characteristics: height (H), collar diameter (DC), stem dry weight (MSPA), root dry weight (MSR), total dry weight (MST), height of the aerial part per collar diameter (H/D), height of the aerial part per aerial dry weight part (H/MSPA), aerial part dry weight per root dry weight (MSPA/MSR) and Dickson Quality Index (IQD), at 120 days after the sown, grown in 'Red Yellow Ultisol'.

\begin{tabular}{|c|c|c|c|c|c|c|c|c|c|c|}
\hline Nutriente & Dose & $\mathrm{H}(\mathrm{cm})$ & $\mathrm{DC}(\mathrm{mm})$ & MSPA (g) & $\operatorname{MSR}(\mathrm{g})$ & $\operatorname{MST}(\mathrm{g})$ & $\mathrm{H} / \mathrm{D}$ & H/MSPA & MSPA/MSR & IQD \\
\hline $\mathrm{N}$ & 0 & 8,48 & 2,71 & 0,33 & 0,47 & 0,79 & 3,22 & 30,04 & 0,69 & 0,22 \\
\hline $\mathrm{N}$ & 50 & 18,90 & 5,40 & 2,86 & 1,90 & 4,76 & 3,53 & 6,80 & 1,51 & 0,96 \\
\hline $\mathrm{N}$ & 100 & 26,25 & 6,52 & 4,40 & 2,66 & 7,06 & 4,03 & 6,01 & 1,68 & 1,25 \\
\hline $\mathrm{N}$ & 150 & 25,80 & 5,65 & 4,16 & 2,25 & 6,41 & 4,57 & 6,77 & 1,79 & 1,00 \\
\hline $\mathrm{N}$ & 200 & 23,83 & 5,70 & 4,58 & 2,49 & 7,07 & 4,17 & 5,47 & 1,80 & 1,18 \\
\hline $\mathrm{P}$ & 0 & 8,48 & 2,71 & 0,33 & 0,47 & 0,79 & 3,22 & 30,04 & 0,69 & 0,22 \\
\hline $\mathrm{P}$ & 150 & 17,38 & 5,22 & 2,67 & 1,85 & 4,52 & 3,36 & 6,68 & 1,44 & 0,96 \\
\hline $\mathrm{P}$ & 300 & 26,25 & 6,52 & 4,40 & 2,66 & 7,06 & 4,03 & 6,01 & 1,68 & 1,25 \\
\hline $\mathrm{P}$ & 450 & 20,62 & 6,31 & 4,10 & 2,71 & 6,82 & 3,32 & 5,32 & 1,51 & 1,44 \\
\hline $\mathrm{P}$ & 600 & 22,35 & 6,15 & 4,48 & 2,76 & 7,24 & 3,61 & 5,16 & 1,61 & 1,37 \\
\hline $\mathrm{K}$ & 0 & 8,48 & 2,71 & 0,33 & 0,47 & 0,79 & 3,22 & 30,04 & 0,69 & 0,22 \\
\hline $\mathrm{K}$ & 50 & 18,43 & 4,74 & 2,66 & 1,47 & 4,13 & 4,19 & 7,61 & 2,06 & 0,76 \\
\hline $\mathrm{K}$ & 100 & 26,25 & 6,52 & 4,40 & 2,66 & 7,06 & 4,03 & 6,01 & 1,68 & 1,25 \\
\hline $\mathrm{K}$ & 150 & 26,90 & 6,89 & 5,78 & 3,06 & 8,84 & 3,95 & 4,76 & 1,87 & 1,53 \\
\hline $\mathrm{K}$ & 200 & 21,23 & 6,80 & 4,67 & 2,56 & 7,23 & 3,15 & 4,89 & 1,81 & 1,47 \\
\hline $\mathrm{Ca}$ & 0 & 8,48 & 2,71 & 0,33 & 0,47 & 0,79 & 3,22 & 30,04 & 0,69 & 0,22 \\
\hline $\mathrm{Ca}$ & 0,8 & 25,58 & 6,69 & 4,97 & 2,81 & 7,78 & 3,82 & 5,22 & 1,76 & 1,39 \\
\hline $\mathrm{Ca}$ & 1,0 & 26,25 & 6,52 & 4,40 & 2,66 & 7,06 & 4,03 & 6,01 & 1,68 & 1,25 \\
\hline $\mathrm{Ca}$ & 1,2 & 24,10 & 6,67 & 5,20 & 2,89 & 8,10 & 3,66 & 4,68 & 1,81 & 1,51 \\
\hline $\mathrm{Ca}$ & 1,4 & 26,08 & 7,06 & 5,56 & 2,76 & 8,32 & 3,69 & 4,73 & 2,03 & 1,45 \\
\hline $\mathrm{MG}$ & 0 & 8,48 & 2,71 & 0,33 & 0,47 & 0,79 & 3,22 & 30,04 & 0,69 & 0,22 \\
\hline $\mathrm{MG}$ & 0,2 & 20,78 & 5,84 & 3,48 & 2,32 & 5,79 & 3,59 & 6,12 & 1,54 & 1,17 \\
\hline MG & 0,4 & 26,25 & 6,52 & 4,40 & 2,66 & 7,06 & 4,03 & 6,01 & 1,68 & 1,25 \\
\hline $\mathrm{MG}$ & 0,6 & 27,30 & 6,33 & 4,88 & 2,36 & 7,24 & 4,32 & 5,62 & 2,07 & 1,13 \\
\hline $\mathrm{MG}$ & 0,8 & 19,08 & 6,13 & 3,80 & 2,49 & 6,30 & 3,14 & 5,21 & 1,53 & 1,37 \\
\hline $\mathrm{S}$ & 0 & 8,48 & 2,71 & 0,33 & 0,47 & 0,79 & 3,22 & 30,04 & 0,69 & 0,22 \\
\hline S & 20 & 19,25 & 5,93 & 3,65 & 2,10 & 5,75 & 3,42 & 6,75 & 1,72 & 1,20 \\
\hline S & 40 & 26,25 & 6,52 & 4,40 & 2,66 & 7,06 & 4,03 & 6,01 & 1,68 & 1,25 \\
\hline $\mathrm{S}$ & 60 & 24,68 & 6,66 & 5,43 & 3,04 & 8,47 & 3,72 & 4,57 & 1,82 & 1,56 \\
\hline $\mathrm{S}$ & 80 & 21,47 & 6,46 & 3,83 & 2,39 & 6,22 & 3,27 & 5,52 & 1,61 & 1,30 \\
\hline
\end{tabular}




\section{Efeito do nitrogênio}

A aplicação de doses crescentes de $\mathrm{N}$ promoveu efeitos que resultaram em modelo linear ou quadrático, exceto para as variáveis DC, MSR, MST, H/MSPA e o índice IQD, para os quais o $\mathrm{N}$ não exerceu efeito significativo (Tabela 4). O modelo foi linear positivo para MSPA e H/D e negativo para MSPA/MSR. Já para $\mathrm{H}$ o efeito foi de ordem raiz quadrada, sendo recomendada a dose de $130,2 \mathrm{mg} / \mathrm{dm}^{3}$ de N. No entanto, deve ficar claro que para a recomendação de adubação não deve ser levada em consideração apenas uma característica isoladamente, estas devem ser analisadas em conjunto visando uma recomendação mais precisa. Desta forma, para a produção de mudas de canafístula de qualidade recomendam-se estudos adicionais com doses inferiores às menores doses testadas, tendo em vista que maior produção será alcançada com a aplicação de no, máximo $50 \mathrm{mg}$ $\mathrm{dm}^{-3}$ de $\mathrm{N}$, ou seja, a dose ótima de $\mathrm{N}$ está entre a dose zero (concentração original do solo) e a menor dose testada.

Efeitos no crescimento de plantas em razão da aplicação de $\mathrm{N}$ são esperados, tendo em vista as diversas funções que este nutriente exerce dentro delas. Efeitos positivos da adubação nitrogenada sobre a produção de diversas espécies florestais arbóreas são encontrados com frequência na literatura. Respostas positivas à aplicação de $\mathrm{N}$ foram observadas por Marques et al. (2006a,b, 2009), Cruz et al. (2006), Ribeiro et al. (2008) e Gonçalves et al. (2008) em plantas de Mimosa caesalpinaefolia, Dalbergia nigra, Piptadenia gonoacantha, Samanea inopinata, Ilex paraguariensis e Anadenathera macrocarpa, respectivamente.

A dose recomendada para a canafístula é inferior à dose recomendada para diversas espécies florestais como Acacia mangium (DIAS et al., 1991), Sclerolobium paniculatum (DIAS et al., 1992), Mimosa caesalpinaefolia, Dalbergia nigra, Piptadenia gonoacantha (MARQUES et al., 2006a,b, 2009, respectivamente) e Samanea inopinata (CRUZ et al., 2006). Apesar dos teores de $\mathrm{N}$ no solo ser originalmente baixo, pode-se afirmar pelas respostas obtidas que a espécie estudada apresenta baixa exigência por $\mathrm{N}$ e que os teores originais deste nutriente no solo encontravam-se próximos do ideal para a canafístula, ou seja, a dose ideal está entre o teor original do solo e a menos dose testada. Pequeno efeito da adição de $\mathrm{N}$ também

TABELA 4: Estimativas geradas para o efeito do $\mathrm{N}\left(\mathrm{mg} \mathrm{dm}^{-3}\right)$, em mudas de canafístula aos 120 dias após a semeadura, cultivadas em Argissolo Vermelho Amarelo, considerando: altura (H), diâmetro do coleto (DC), peso de matéria seca da parte aérea (MSPA), peso de matéria seca de raiz (MSR), peso de matéria seca total (MST), altura de parte aérea por diâmetro do coleto (H/D), altura de parte aérea por peso de matéria seca de parte aérea (H/MSPA), peso de matéria seca de parte aérea por peso de matéria seca de raiz (MSPA/MSR) e Índice de Qualidade de Dickson (IQD).

TABLE 4: Generated estimations from the effect of $\mathrm{N}\left(\mathrm{mg} \mathrm{dm}^{-3}\right)$, in Peltophorum dubium seedlings at 120 days after sown, grown in 'Red Yellow Ultisol', considering: height $(\mathrm{H})$, collar diameter (DC), aerial part dry weight (MSPA), root dry weight (MSR), total dry weight (MST), height of the aerial part per collar diameter (H/D), height of the aerial part per aerial part dry weight (H/MSPA), aerial part dry weight per root dry weight (MSPA/MSR) and Dickson Quality Index (IQD).

\begin{tabular}{llccc}
\hline Característica & \multicolumn{1}{c}{ Equação } & $\begin{array}{c}\text { Significância } \\
(\%)\end{array}$ & $\mathrm{R}^{2}$ & $\begin{array}{c}\text { Dose } \\
\text { recomendada }\end{array}$ \\
\hline H & $\hat{Y}=-25,9661+9,2157 \sqrt{X}-0,4038 X$ & 5 & 96,50 & 130,2 \\
DC & $\hat{Y}=\bar{Y}=5,82$ & & & 50 \\
MSPA & $\hat{Y}=2,7675+0,0098 X$ & 10 & 66,16 & 200 \\
MSR & $\hat{Y}=\bar{Y}=2,33$ & & & 50 \\
MST & $\hat{Y}=\bar{Y}=6,35$ & & & 50 \\
H/D & $\hat{Y}=\bar{Y}=3,4581+0,0049 X$ & 5 & 55,24 & 50 \\
H/MSPA & $\hat{Y}=\bar{Y}=6,26$ & & & 50 \\
MSPA/MSR & $\hat{Y}=1,4483+0,002 X$ & 5 & 89,01 & 50 \\
IQD & $\hat{Y}=\bar{Y}=1,10$ & & & 50 \\
\hline
\end{tabular}

Ci. Fl., v. 21, n. 3, jul.-set., 2011 
foi verificado por Otto et al. (2007) e Ribeiro et al. (2008) em plantas de Populus deltoides e Ilex paraguariensis, respectivamente.

\section{Efeito do fósforo}

$\mathrm{O} \mathrm{P}$ foi o nutriente que promoveu mais efeitos significativos no crescimento e qualidade das mudas de canafístula. Os efeitos gerados foram de ordem raiz quadrada para $\mathrm{H}$ e DC (Tabela 5), em que o aumento das doses favoreceu o incremento dos valores dessas características até um ponto de máximo, nas doses 376,8 e $397,2 \mathrm{mg} / \mathrm{dm}^{3}$ de $\mathrm{P}$, respectivamente, quando então diminuíram. As variáveis MSPA, MSR, MST e IQD foram descritas por modelo linear positivo e a relação H/MSPA apresentou reposta oposta, ou seja, efeito linear negativo (Tabela 5), indicando que maior produção será alcançada com a aplicação de doses superiores a $600 \mathrm{mg} / \mathrm{dm}^{3}$ de $\mathrm{P}$.

As doses recomendadas para canafístula são superiores às encontradas por Gonçalves et al. (2008) para Anadenanthera macrocarpa, em condições experimentais semelhantes e utilizando o mesmo tipo de solo, fonte e doses dos fertilizantes fosfatados.
Outros autores também verificaram bom crescimento de mudas cultivadas com dose de $\mathrm{P}$ inferior à recomendada neste estudo. Ceconi et al. (2006) recomendam a aplicação de $360 \mathrm{mg} \mathrm{dm}^{-3} \mathrm{de} P$ na produção de mudas de Luehea divaricata; Gomes et al. (2008) recomendam $54 \mathrm{mg} \mathrm{dm}^{-3}$ de P para Apuleia leiocarpa; Ceconi et al. (2007) recomendam $450 \mathrm{mg} \mathrm{dm}^{-3}$ de $\mathrm{P}$ para Ilex paraguariensis. Isto indica que a espécie estudada mostra-se mais exigente em $\mathrm{P}$, o que também foi observado por Resende et al. (1999), Mello et al. (2008) e Lima et al. (2008), os quais recomendaram aplicação de doses de $\mathrm{P}$ semelhantes ou até mesmo superiores às indicadas para canafístula, na produção de mudas de Schinus terebinthifolius, Acacia mearnsii e Euterpe edulis, respectivamente.

A importância da fertilização fosfatada na produção de mudas de canafístula também foi evidenciada por Venturin et al. (1999), os quais destacaram que a presença de $\mathrm{P}$ no meio de crescimento é fundamental para o crescimento satisfatório da espécie, observando que a mesma é muito exigente em $\mathrm{P}$, e a sua ausência é prejudicial ao adequado crescimento e bom padrão de qualidade das mudas.

TABELA 5: Estimativas geradas para o efeito do $\mathrm{P}\left(\mathrm{mg} \mathrm{dm}^{-3}\right)$, em mudas de canafístula aos 120 dias após a semeadura, cultivadas em Argissolo Vermelho Amarelo, considerando: altura (H), diâmetro do coleto (DC), peso de matéria seca da parte aérea (MSPA), peso de matéria seca de raiz (MSR), peso de matéria seca total (MST), altura de parte aérea por diâmetro do coleto (H/D), altura de parte aérea por peso de matéria seca de parte aérea (H/MSPA), peso de matéria seca de parte aérea por peso de matéria seca de raiz (MSPA/MSR) e Índice de Qualidade de Dickson (IQD).

TABLE 5: Generated estimations from the effect of $\mathrm{P}\left(\mathrm{mg} \mathrm{dm}^{-3}\right)$, in Peltophorum dubium seedlings at 120 days after sown, grown in Red Yellow Ultisol, considering: height $(\mathrm{H})$, collar diameter (DC), aerial part dry weight (MSPA), root dry weight (MSR), total dry weight (MST), height of the aerial part per collar diameter (H/D), height of the aerial part per aerial part dry weight (H/MSPA), aerial part dry weight per root dry weight (MSPA/MSR) and Dickson Quality Index (IQD).

\begin{tabular}{llccc}
\hline Característica & \multicolumn{1}{c}{ Equação } & $\begin{array}{c}\text { Significância } \\
\text { (\%) }\end{array}$ & $\mathrm{R}^{2}$ & $\begin{array}{c}\text { Dose } \\
\text { recomendada }\end{array}$ \\
\hline H & $\hat{Y}=-25,9961+9,2157 \sqrt{X}-0,4038 X$ & 5 & 49,38 & 376,8 \\
DC & $\hat{Y}=-1,9965+0,8549 \sqrt{X}-0,0214 X$ & 10 & 90,29 & 397,2 \\
MSPA & $\hat{Y}=2,6275+0,0034 X$ & 10 & 61,33 & 600 \\
MSR & $\hat{Y}=1,7975+0,0018 X$ & 5 & 69,47 & 600 \\
MST & $\hat{Y}=4,425+0,0053 X$ & 5 & 64,61 & 600 \\
H/D & $\hat{Y}=\bar{Y}=3,58$ & & & 150 \\
H/MSPA & $\hat{Y}=7,1059-0,0035 X$ & 5 & 94,66 & 600 \\
MSPA/MSR & $\hat{Y}=\bar{Y}=1,56$ & & & 150 \\
IQD & $\hat{Y}=0,9004+0,0009 X$ & 5 & 75,70 & 600 \\
\hline
\end{tabular}


Fernandes et al. (2002 e 2007) também observaram efeito linear positivo, na produção de matéria seca, da aplicação de P até uma dosagem de $450 \mathrm{mg} \mathrm{dm}^{-3} \mathrm{em}$ mudas de Cordia goeldiana. Deve-se ressaltar, entretanto, que as plantas de canafístula apresentaram este comportamento até uma dose superior à maior dose aplicada pelos autores anteriores, demonstrando, desta forma, ser mais exigente nutricionalmente em $\mathrm{P}$ do que Cordia goeldiana.

\section{Efeito do potássio}

À exceção da relação MSPA/MSR, a aplicação de doses crescentes de $\mathrm{K}$ proporcionou efeitos que resultaram em modelo linear e de ordem raiz quadrada, de acordo com a característica em questão (Tabela 6).

$\mathrm{O}$ efeito da aplicação de $\mathrm{K}$ foi de ordem raiz quadrada para $\mathrm{H}$, MSPA, MSR e MST em que o aumento das doses favoreceu o aumento dessas variáveis até um ponto de máximo, nas doses de 120,$7 ; 147,5 ; 139,7$ e $144,4 \mathrm{mg} / \mathrm{dm}^{3}$ de $\mathrm{K}$, respectivamente.

Para DC e o índice IQD o efeito foi explicado por modelo linear positivo. Em contrapartida, para as relações H/D e H/MSPA ocorreu efeito linear negativo. Em ambos os casos, tanto do efeito positivo ou negativo, nessas condições, recomendase a aplicação de doses superiores a $200 \mathrm{mg} / \mathrm{dm}^{3}$ de $\mathrm{K}$, visando maior crescimento e qualidade das mudas de canafístula. Os resultados obtidos com a aplicação de doses de $\mathrm{K}$ indicam que a espécie estudada apresenta alta exigência para esse nutriente, quando cultivada neste tipo de substrato, sendo necessária a fertilização do substrato para a obtenção de mudas com padrão de qualidade satisfatório.

Diversos autores constataram ausência de efeito da aplicação de $\mathrm{K}$ sobre o crescimento e qualidade das mudas, o que é mais um indicativo de que a canafístula é uma espécie exigente para este nutriente. Baliero et al. (2001) verificaram melhor desempenho de mudas de Acacia auriculiformis cultivadas sem a aplicação de $\mathrm{K}$, o mesmo foi observado por Duboc et al. (1996), Souza et al. (2006) e Gonçalves et al. (2008) em mudas de Hymenaea courbaril, Tabebuia impetiginosa e Anadenathera macrocarpa, respectivamente.

De maneira semelhante ao observado neste estudo, Dias et al. (1992) também observaram

TABELA 6: Estimativas geradas para o efeito do $\mathrm{K}\left(\mathrm{mg} \mathrm{dm}^{-3}\right)$, em mudas de canafístula aos 120 dias após a semeadura, cultivadas em Argissolo Vermelho Amarelo, considerando: altura (H), diâmetro do coleto (DC), peso de matéria seca da parte aérea (MSPA), peso de matéria seca de raiz (MSR), peso de matéria seca total (MST), altura de parte aérea por diâmetro do coleto (H/D), altura de parte aérea por peso de matéria seca de parte aérea (H/MSPA), peso de matéria seca de parte aérea por peso de matéria seca de raiz (MSPA/MSR) e Índice de Qualidade de Dickson (IQD).

TABLE 6: Generated estimations from the effect of $\mathrm{K}\left(\mathrm{mg} \mathrm{dm}^{-3}\right)$, in Peltophorum dubium seedlings at 120 days after sown, grown in Red Yellow Ultisol, considering: height $(\mathrm{H})$, collar diameter (DC), aerial part dry weight (MSPA), root dry weight (MSR), total dry weight (MST), height of the aerial part per collar diameter (H/D), height of the aerial part per aerial part dry weight (H/MSPA), aerial part dry weight per root dry weight (MSPA/MSR) and Dickson Quality Index (IQD).

\begin{tabular}{llccc}
\hline Característica & \multicolumn{1}{c}{ Equação } & $\begin{array}{c}\text { Significância } \\
(\%)\end{array}$ & $\mathrm{R}^{2}$ & $\begin{array}{c}\text { Dose } \\
\text { recomendada }\end{array}$ \\
\hline H & $\hat{Y}=-43,2910+12,8457 \sqrt{X}-0,5846 X$ & 1 & 99,99 & 120,7 \\
DC & $\hat{Y}=4,6063+0,0130 X$ & 10 & 69,71 & 200 \\
MSPA & $\hat{Y}=-10,3573+2,5765 \sqrt{X}-0,1061 X$ & 5 & 97,55 & 147,5 \\
MSR & $\hat{Y}=-6.4671+1.5967 \sqrt{X}-0.0676 X$ & 1 & 99,97 & 139,7 \\
MST & $\hat{Y}=-16,8244+4,1731 \sqrt{X}-0,1736 X$ & 5 & 98,64 & 144,4 \\
H/D & $\hat{Y}=4,6247-0,0063 X$ & 5 & 79,16 & 200 \\
H/MSPA & $\hat{Y}=8,1729-0,0189 X$ & 1 & 84,84 & 200 \\
MSPA/MSR & $\hat{Y}=\bar{Y}=1,86$ & & & 50 \\
IQD & $\hat{Y}=0,6454+0,0048 X$ & 10 & 79,59 & 200 \\
\hline
\end{tabular}

Ci. Fl., v. 21, n. 3, jul.-set., 2011 
resposta positiva das mudas de Sclerolobium paniculatum à adubação potássica. A dose de $\mathrm{K}$ recomendada para canafístula é bem maior do que a recomendada por Reis et al. (1997) para mudas de Dalbergia nigra, estando esse autor de acordo com relatos de Dias et al. (1991), que menciona que a maioria das espécies florestais exige a dose de $50 \mathrm{mg} / \mathrm{dm}^{3}$ de $\mathrm{K}$. Isto implica que a espécie em estudo é altamente exigente em $\mathrm{K}$, corroborando com o estudo de Venturin et al. (1999), os quais concluíram que a canafístula apresenta elevada exigência nutricional para este nutriente.

\section{Efeito do cálcio}

Em razão da pouca variação nos valores observados, o $\mathrm{Ca}$ foi o nutriente que gerou menos efeitos significativos nas diversas características estudadas (Tabela 7), os quais foram obtidos nas relações H/MSPA e MSPA/MSR, ambos descritos por modelo linear, sendo o primeiro com efeito negativo e o outro positivo. A recomendação, para as características com efeito significativo, é que devem ser aplicadas doses maiores que $1,4 \mathrm{cmol} /$ $\mathrm{dm}^{3}$.

A ausência de efeitos significativos pode estar relacionada aos teores de Ca que esse solo apresentava originalmente $\left(1,60 \mathrm{cmol}_{\mathrm{c}} \mathrm{dm}^{-3}\right)$. Venturin et al. (1999) observaram resposta contrária à verificada neste estudo com a mesma espécie, ou seja, os autores obtiveram efeito significativo da aplicação de Ca sobre o crescimento das mudas de canafístula. No entanto, o solo usado como substrato neste estudo apresentava teores originais do nutriente bem superiores aos encontrados no substrato usado pelos autores mencionados, sendo a diferença de $1,46 \mathrm{cmol}_{\mathrm{c}} \mathrm{dm}^{-3} \mathrm{de}$ Ca entre o solo usado neste estudo e o solo usado no estudo de Venturin et al. (1999). Além disso, esses autores testaram a elevação dos teores de Ca no solo até $0,49 \mathrm{cmol}_{\mathrm{c}} \mathrm{dm}^{-}$ ${ }^{3}$, sendo esta dosagem inferior a menor dose testada no experimento em questão, o que pode explicar a divergência de resultados encontrada.

Em estudos de outros autores, essa falta de resposta em razão da aplicação de Ca também foi verificada, como em mudas de Hymenaea courbaril e Dalbergia nigra, estudadas por DUBOC et al. (1996) e Reis et al. (1997), respectivamente.

Entretanto, o efeito da adição de Ca já foi observado em outras espécies, cultivadas em substratos com teores de $\mathrm{Ca}$ superiores a

TABELA 7: Estimativas geradas para o efeito do $\mathrm{Ca}\left(\mathrm{cmol}_{\mathrm{c}} \mathrm{dm}^{-3}\right)$, em mudas de canafístula aos 120 dias após a semeadura, cultivadas em Argissolo Vermelho Amarelo, considerando: altura (H), diâmetro do coleto (DC), peso de matéria seca da parte aérea (MSPA), peso de matéria seca de raiz (MSR), peso de matéria seca total (MST), altura de parte aérea por diâmetro do coleto (H/D), altura de parte aérea por peso de matéria seca de parte aérea (H/MSPA), peso de matéria seca de parte aérea por peso de matéria seca de raiz (MSPA/MSR) e Índice de Qualidade de Dickson (IQD).

TABLE 7: Generated estimates from the effect of $\mathrm{Ca}\left(\mathrm{cmol}_{\mathrm{c}} \mathrm{dm}^{-3}\right)$, in Peltophorum dubium seedlings at 120 days after sown, grown in 'Red Yellow Ultisol', considering: height $(\mathrm{H})$, collar diameter (DC), aerial part dry weight (MSPA), root dry weight (MSR), total dry weight (MST), height of the aerial part per collar diameter (H/D), height of the aerial part per aerial part dry weight (H/MSPA), aerial part dry weight per root dry weight (MSPA/MSR) and Dickson Quality Index (IQD).

\begin{tabular}{|c|c|c|c|c|}
\hline Característica & Equação & Significância (\%) & $\mathrm{R}^{2}$ & Dose recomendada \\
\hline $\mathrm{H}$ & $\hat{Y}=\bar{Y}=23,20$ & & & 0,8 \\
\hline DC & $\hat{Y}=\bar{Y}=6,24$ & & & 0,8 \\
\hline MSPA & $\hat{Y}=\bar{Y}=4,38$ & & & 0,8 \\
\hline MSR & $\hat{Y}=\bar{Y}=2,44$ & & & 0,8 \\
\hline MST & $\hat{Y}=\bar{Y}=6,82$ & & & 0,8 \\
\hline $\mathrm{H} / \mathrm{D}$ & $\hat{Y}=\bar{Y}=3,83$ & & & 0,8 \\
\hline H/MSPA & $\hat{Y}=6,6966-1,3965 X$ & 5 & 34,15 & 1,4 \\
\hline MSPA/MSR & $\hat{Y}=1,3026+0,4692 X$ & 5 & 65,50 & 1,49 \\
\hline IQD & $\hat{Y}=\bar{Y}=1,25$ & & & 0,8 \\
\hline
\end{tabular}


0,2 $\mathrm{cmol}_{\mathrm{c}} / \mathrm{dm}^{3}$, como em mudas de Tabebuia impetiginosa, Samanea inopinata, Dalbergia nigra, Machaerium nictitans e Apuleia leiocarpa submetidas à elevação de saturação por bases e usando argissolo como substrato Cruz et al. (2004, 2006), Bernardino et al. (2007), Souza et al. (2008) e Gomes et al. (2008), respectivamente.

\section{Efeito do magnésio}

O crescimento das plantas sob condições diferenciadas de $\mathrm{Mg}$ foi significativo para todas as características estudadas, exceto para DC, MSR e o índice IQD (Tabela 8). Os efeitos resultaram em modelos linear, quadrático e raiz quadrada, de acordo com a característica em questão.

$\mathrm{O}$ efeito da aplicação de $\mathrm{N}$ foi quadrático para as variáveis $\mathrm{H}, \mathrm{H} / \mathrm{D}$ e MSPA/MSR e de ordem raiz quadrada para MSPA e MST, em que o aumento das doses de $\mathrm{Mg}$ favoreceu a elevação dos valores das características até um ponto de máximo, em média na dosagem de $0,50 \mathrm{cmol}_{\mathrm{c}} / \mathrm{dm}^{3}$ de $\mathrm{Mg}$, quando então, as médias diminuíram. Para a relação H/MSPA foi verificado efeito linear negativo, como quanto menor o valor desse índice melhor o padrão de qualidade da muda produzida, recomenda-se a aplicação de doses superiores a $0,8 \mathrm{cmol} / \mathrm{cm}^{3} \mathrm{de}$ $\mathrm{Mg}$.

Venturin et al. (1999) observaram também para canafístula que o $\mathrm{Mg}$ se mostrou limitante ao crescimento das plantas, corroborando com o obtido no presente estudo. Além disso, efeitos da aplicação de $\mathrm{Mg}$ são encontrados com frequência na literatura para diversas espécies florestais, como Myracrodruon urundeuva, Tabebuia impetiginosa, Samanea inopinata, Dalbergia nigra, Machaerium nictitans e Apuleia leiocarpa estudada por Mendonça et al. (1999), Cruz et al. (2004, 2006), Bernardino et al. (2007), Souza et al. (2008) e Gomes et al. (2008), respectivamente.

\section{Efeito do enxofre}

Verificou-se efeito significativo da adição de doses de S para as características H, MSPA, MSR e MST, sendo descrito por modelos quadrático ou raiz quadrada, nas quais o ponto de máximo foi obtido em doses que variaram de 47,27 a $53,7 \mathrm{mg} /$ $\mathrm{dm}^{3}$ (Tabela 9).

Contrariando o verificado para canafístula, Reis et al. (1997) observaram para Dalbergia nigra resposta negativa à aplicação de enxofre,

TABELA 8: Estimativas geradas para o efeito do $\mathrm{Mg}\left(\mathrm{cmol}_{\mathrm{c}} \mathrm{dm}^{-3}\right)$, em mudas de canafístula aos 120 dias após a semeadura, cultivadas em Argissolo Vermelho Amarelo, considerando: altura (H), diâmetro do coleto (DC), peso de matéria seca da parte aérea (MSPA), peso de matéria seca de raiz (MSR), peso de matéria seca total (MST), altura de parte aérea por diâmetro do coleto (H/D), altura de parte aérea por peso de matéria seca de parte aérea (H/MSPA), peso de matéria seca de parte aérea por peso de matéria seca de raiz (MSPA/MSR) e Índice de Qualidade de Dickson (IQD).

TABLE 8: Generated estimates from the effect of $\mathrm{Mg}\left(\mathrm{cmol}_{\mathrm{c}} \mathrm{dm}^{-3}\right)$, in Peltophorum dubium seedlings at 120 days after sown, grown in 'Red Yellow Ultisol', considering: height $(\mathrm{H})$, collar diameter (DC), aerial part dry weight (MSPA), root dry weight (MSR), total dry weight (MST), height of the aerial part per collar diameter (H/D), height of the aerial part per aerial part dry weight (H/MSPA), aerial part dry weight per root dry weight (MSPA/MSR) and Dickson Quality Index (IQD).

\begin{tabular}{llccc}
\hline Característica & \multicolumn{1}{c}{ Equação } & $\begin{array}{c}\text { Significância } \\
(\%)\end{array}$ & $\mathrm{R}^{2}$ & $\begin{array}{c}\text { Dose } \\
\text { recomendada }\end{array}$ \\
\hline H & $\hat{Y}=-85,625 X^{2}+83,6 X+7,2375$ & 1 & 97,60 & 0,49 \\
DC & $\hat{Y}=\bar{Y}=6,21$ & & & 0,2 \\
MSPA & $\hat{Y}=-5,3781+28,9526 \sqrt{X}-20,7535 X$ & 5 & 95,76 & 0,49 \\
MSR & $\hat{Y}=\bar{Y}=2,46$ & & & 0,2 \\
MST & $\hat{Y}=-4,2885+33,0884 \sqrt{X}-23,7056 X$ & 10 & 99,99 & 0,49 \\
H/D & $\hat{Y}=-10.1045 X^{2}+9.5756 X+2.0135$ & 1 & 89,12 & 0,2 \\
H/MSPA & $\hat{Y}=6,5240-1,5676 X$ & 5 & 95,15 & 0,8 \\
MSPA/MSR & $\hat{Y}=-4,2802 X^{2}+4,4602 X+0,7593$ & 1 & 64,28 & 0,52 \\
IQD & $\hat{Y}=\bar{Y}=1,23$ & & & 0,2 \\
\hline
\end{tabular}

Ci. Fl., v. 21, n. 3, jul.-set., 2011 
TABELA 9: Estimativas geradas para o efeito do $\mathrm{S}\left(\mathrm{mg} \mathrm{dm}^{-3}\right)$, em mudas canafístula aos 120 dias após a semeadura, cultivadas em Argissolo Vermelho Amarelo, considerando: altura (H), diâmetro do coleto (DC), peso de matéria seca da parte aérea (MSPA), peso de matéria seca de raiz (MSR), peso de matéria seca total (MST), altura de parte aérea por diâmetro do coleto (H/D), altura de parte aérea por peso de matéria seca de parte aérea (H/MSPA), peso de matéria seca de parte aérea por peso de matéria seca de raiz (MSPA/MSR) e Índice de Qualidade de Dickson (IQD).

TABLE 9: Generated estimations from the effect of $\mathrm{S}\left(\mathrm{mg} \mathrm{dm}^{-3}\right)$, in Peltophorum dubium seedlings at 120 days after sown, grown in Red Yellow Ultisol, considering: height $(\mathrm{H})$, collar diameter (DC), aerial part dry weight (MSPA), root dry weight (MSR), total dry weight (MST), height of the aerial part per collar diameter (H/D), height of the aerial part per aerial part dry weight (H/MSPA), aerial part dry weight per root dry weight (MSPA/MSR) and Dickson Quality Index (IQD).

\begin{tabular}{llccc}
\hline Característica & \multicolumn{1}{c}{ Equação } & $\begin{array}{c}\text { Significância } \\
(\%)\end{array}$ & $\mathrm{R}^{2}$ & $\begin{array}{c}\text { Dose } \\
\text { recomendada }\end{array}$ \\
\hline H & $\hat{Y}=-29,283+16,1222 \sqrt{X}-1,1725 X$ & 1 & 95,26 & 47,27 \\
DC & $\hat{Y}=\bar{Y}=6,39$ & & & 20 \\
MSPA & $\hat{Y}=-0,0014 X^{2}+0,1523 X+1,0319$ & 5 & 78,64 & 53,0 \\
MSR & $\hat{Y}=-3,0079+1,6496 \sqrt{X}-0,1153 X$ & 10 & 95,89 & 51,1 \\
MST & $\hat{Y}=-0,0022 X^{2}+0,2314 X+1,8$ & 5 & 83,98 & 53,7 \\
H/D & $\hat{Y}=\bar{Y}=3,61$ & & & 20 \\
H/MSPA & $\hat{Y}=\bar{Y}=5,71$ & & & 20 \\
MSPA/MSR & $\hat{Y}=\bar{Y}=1,71$ & & & 20 \\
IQD & $\hat{Y}=\bar{Y}=1,33$ & & 20 \\
\hline
\end{tabular}

evidenciando que o teor do nutriente original do solo $\left(4,8 \mathrm{mg} \mathrm{dm}^{-3}\right)$ seria superior ao exigido pela espécie. Em contrapartida, Balieiro et al. (2001) constataram, para mudas de Acacia holosericea, uma resposta positiva à aplicação de enxofre ao substrato, assim como Fernández et al. (1996) em mudas de Mimosa tenuiflora.

\section{CONCLUSÕES}

A aplicação de fertilizantes à base de macronutrientes, comparados com a não adição de nutrientes, levou a ganhos significativos no crescimento e qualidade das mudas de canafístula. No entanto, para alguns nutrientes estudados como $\mathrm{N}$ e Ca, o efeito observado da aplicação de doses crescentes dos mesmos foi pequeno, ao contrário do verificado para os demais nutrientes.

Assim, de acordo com os resultados deste trabalho, recomendam-se estudos adicionais com os nutrientes $\mathrm{Ne} \mathrm{Ca}$, com doses inferiores às menores doses testadas, tendo em vista que possivelmente a melhor dose destes nutrientes, a ser recomendada, encontra-se entre os teores originais destes nutrientes no solo usado como substrato e a menor dose testada.

Com base nos resultados obtidos recomenda-se a aplicação de $600 \mathrm{mg} \mathrm{dm}^{-3}$ de $\mathrm{P}, 150$ $\mathrm{mg} \mathrm{dm}{ }^{-3} \mathrm{de} \mathrm{K}, 0,50 \mathrm{cmol}_{\mathrm{c}} \mathrm{dm}^{-3} \mathrm{de} \mathrm{Mg}$ e $50 \mathrm{mg} \mathrm{dm}^{-3}$ de S, utilizando Argissolo Vermelho Amarelo como substrato.

\section{AGRADECIMENTOS}

Os autores agradecem ao $\mathrm{CNPq}$, pela concessão da bolsa de estudos e pela bolsa de produtividade em pesquisa, e ao Projeto PRODETAB 130-02/01, pelo financiamento do presente trabalho.

\section{REFERÊNCIAS BIBLIOGRÁFICAS}

ALVAREZ V., V. H. Equilíbrio de formas disponíveis de fósforo e enxofre em dois Latossolos de Minas Gerais. 1974. $125 \mathrm{f}$. Dissertação (Mestrado em Solos e Nutrição de Plantas)-Universidade Federal de Viçosa, Viçosa, 1974.

BALIEIRO, F. C. et al. Formação de mudas de Acacia holosericea e Acacia auriculiformis: Resposta à calagem, fósforo, potássio e enxofre. 
Revista Árvore, Viçosa, v. 25, n. 2, p. 183-191, mar./abr.2001.

BARBERI; A. et al. Nodulação de leguminosas florestais em viveiros no sul Minas Gerais. Cerne, Lavras, v. 4, n. 1, p. 145-153, jan./mar. 1998.

BERNARDINO, D. C. de S. et al. Influência da saturação por bases e da relação Ca:Mg do substrato sobre o crescimento inicial de jacarandá-da-bahia (Dalbergia nigra (Vell.) Fr. All. Ex Benth.). Revista Árvore, Viçosa, v. 31, n. 4, p. 567-573, jul./ago. 2007.

CARNEIRO, J. G. A. Variações na metodologia de produção de mudas florestais afetam os parâmetros morfofisiológicos que indicam a sua qualidade. Série Técnica FUPEF, n. 12, p. 1-40, 1983.

CARneIRO, J. G. A. Produção e controle de qualidade de mudas florestais. Curitiba: UFPR/ FUPEF, 1995. $451 \mathrm{p}$.

CARVALHO, M. M. Ações visando recuperação de áreas de pastagens degradadas. In: ENCONTRO PARA CONSERVAÇÃO DA NATUREZA, 1., 1997, Viçosa. Anais... Viçosa: CMCN/DEF/UFV, 1997. p. 202-206.

CARVALHO, P. E. R. Espécies arbóreas brasileiras. Colombo: EMBRAPA Florestas, 2003, v.1. 1039 p.

CECONI, D. E. et al. Crescimento de mudas de açoita-calavo (Luehea divaricata Mart.) sob influência da adubação fosfatada. Cerne, Lavras, v. 12, n. 3, p. 292-299, jul./set. 2006.

CECONI, D. E. et al. Exigência nutricional de mudas de erva-mate (Ilex paraguariensis A. St.Hil.) à adubação fosfatada. Ciência Florestal, Santa Maria, v. 17, n. 1, p. 25-32, jan./abr. 2007.

CHAVES, L. de L. B. et al. Crescimento de mudas de Andenathera macrocarpa (Benth) Brenan (angicovermelho) em substrato fertilizado e inoculado com rizóbio. Revista Árvore, v. 30, n. 6, p. 911-919, nov./dez. 2006.

COELHO, S. R. de F. et al. Crescimento, nutrição e fixação biológica de nitrogênio em plantios mistos de eucalipto e leguminosas arbóreas. Pesquisa Agropecuária Brasileira, Brasília, v. 42, n. 6, p. 759-768, jun. 2007.

CRUZ, C. A. F. et al. Efeito de diferentes níveis de saturação por bases no desenvolvimento e qualidade de mudas de ipê-roxo (Tabebuia impetiginosa (Mart.) Standley). Scientia Forestalis, Piracicaba, n. 66, p. 100-107, set./dez. 2004.

CRUZ, C. A. F. et al. Efeito da adubação nitrogenada na produção de mudas de sete-cascas (Samanea inopinata (Harms) Ducke) Revista Árvore, Viçosa, v. 30, n. 4, p. 537-546, jul./ago. 2006.

DIAS, L. E. et al. Formação de mudas de Acacia mangium Willd: 2. Resposta a nitrogênio e potássio. Revista Árvore, Viçosa, v. 15, n.1, p. 11-22, jan./ fev. 1991.

DIAS, L. E. et al. Formação de mudas de táxi-branco (Sclerolobium paniculatum Voguel): II. Resposta a nitrogênio, potássio e enxofre. Revista Árvore, Viçosa, v. 16, n. 2, p. 135-143, mar./abr. 1992.

DIAS, P. F. et al. Transferência do N fixado por leguminosas arbóreas para o capim Survelona crescido em consórcio. Ciência Rural, Santa Maria, v. 37, n. 2, p. 352-356, mar./abr. 2007.

DICKSON, A. et al.. Quality appraisal of white spruce and white pine seedling stock in nurseries. Forestry Chronicle, v. 36, p. 10-13, 1960.

DUBOC, E. et al. Nutrição do jatobá (Hymenaea courbaril L. var. Stilbocarpa (Hayne) Lee et Lang.). Cerne, Lavras, v. 2, n. 1, p. 31-47, jan./mar. 1996. EUCLYDES, R. F. Manual de utilização do programa SAEG (Sistema para análises estatísticas e genéticas), Viçosa, MG: UFV, 1997. $59 \mathrm{p}$.

FERNANDES, A. R. et al. Efeito do fósforo e do zinco sobre o crescimento de mudas de freijó (Cordia goeldiana Huber.). Revista de Ciências Agrárias, Belém, n. 37, p. 123-131, jan./jun. 2002. FERNANDES, A. R. et al. Crescimento e absorção de nutrientes por mudas de freijó (Crodia goeldiana Huber) em função de doses de fósforo e zinco. Revista Árvore, Viçosa, v. 31, n. 4, p. 599-608, jul./ ago. 2007.

FERNÁNDEZ, J. Q. P. et al. Crescimento de mudas de Mimosa tenuiflora submetidas a diferentes níveis de calagem e doses de fósforo, potássio e enxofre. Revista Árvore, Viçosa, v. 20, n. 4, p. 425-431, jul./ ago. 1996.

GOMES, K. C. de O. et al. Crescimento de mudas de garapa em reposta à calagem e ao fósforo. Revista Árvore, Viçosa, v. 32, n. 3, p. 387-394, jul./ago. 2008.

GONÇALVES, J. L. M.; BENEDETTI, V. Nutrição e fertilização florestal. Piracicaba: IPEF, 2000. 427 p.

GONÇALVES, E. de O. et al. Crescimento de mudas de angico-vermelho (Anadenanthera macrocarpa (Benth.) Brenan) sob diferentes doses de macronutrientes. Revista Árvore, Viçosa, v. 32, n. 6, p. 1029-1040, nov./dez. 2008.

LORENZI, H. Árvores brasileiras: manual de identificação e cultivo de plantas arbóreas nativas

Ci. Fl., v. 21, n. 3, jul.-set., 2011 
do Brasil. Nova Odessa: Ed. Plantarum, 1992. 352 p. LIMA, L. S. H. et al. Crescimento de mudas de Euterpe edulis Martius em reposta a diferentes doses de fósforo. Ciência Florestal, Santa Maria, v. 18, n. 4, p. 461-470, out./dez. 2008.

MARCHIORI, J. N. C. Dendrologia das angiospermas: leguminosas. Santa Maria: UFSM, 1997. $2000 \mathrm{p}$.

MARQUES, V. B. et al. Efeito de fontes e doses de nitrogênio no crescimento de mudas de sabiá (Mimosa caesalpiniaefolia Benth.). Scientia Forestalis, Piracicaba, n. 71, p. 77-85, maio/ago. $2006 a$.

MARQUES, V. B. et al. Efeito de fontes e doses de nitrogênio sobre o crescimento de inicial e qualidade de mudas de jacarandá-da-bahia (Dalbergia nigra (Vell.) Fr. All. Ex Benth.). Revista Árvore, Viçosa, v. 30, n. 5, p. 725-735, set./out. 2006b.

MARQUES, L. S. et al. Crescimento de mudas de jacaré (Piptadenia gonoacantha J. F. Macbr.) em diferentes tipos de solos e fontes e doses de nitrogênio. Revista Árvore, Viçosa, v. 33, n. 1, p. 81-91, jan./fev. 2009.

MELLO, A. H. de et al. Influência de substratos e fósforo na produção de mudas micorrizadas de Acacia mearnsii de Wild. Ciência Florestal, Santa Maria, v. 18, n. 3, p. 321-327, jul./set. 2008.

MENDONÇA, A. V. R. et al. Exigências nutricionais de Myracroduon urundeuva Fr. All (Aroeira do Sertão). Cerne, Lavras, v. 5, n. 2, p. 65-75, abr./jun. 1999.

OTTO, G. M. et al. Resposta do álamo (Populus deltoides Marsch)à adubação nitrogenada em dois sítios do município de São Mateus do Sul, Paraná.
Ciência Florestal, Santa Maria, v. 17, n. 2, p. 8190, abr./jun. 2007.

RAIJ, B. V. Fertilidade do solo e adubação. Piracicaba: Ceres; POTAFOS, 1991. 343 p.

REIS, M. G. F. et al. Exigências nutricionais de mudas de Dalbergia nigra (Vell.) Fr. Allem (Jacarandá-da-Bahia) produzidas em dois níveis de sombreamento. Revista Árvore, Viçosa, v. 21, n. 4, p. 463-471, jul./ago. 1997.

RESENDE, A. V. et al. Crescimento inicial de espécies florestais de diferentes grupos sucessionais em resposta a doses de fósforo. Pesquisa Agropecuária Brasileira, Brasília, v. 34, n. 11, p. 2071-2081, nov. 1999.

RIBEIRO, M. M. et al. Nutrição da erva-mate com sulfato de amônio. Cerne, Lavras, v. 14, n. 3, p. 204-211, jul./set. 2008.

SOUZA, C. A. M. de et al. Crescimento em campo de espécies florestais em diferentes condições de adubações. Ciência Florestal, Santa Maria, v.16, n. 3, p. 243-249, jul./set. 2006.

SOUZA, P. H. de et al. Influência da saturação por bases do substrato no crescimento e qualidade de mudas de Machaerium nictitans (Vell.) Benth. Revista Árvore, Viçosa, v. 32, n. 2, p. 193-201, mar./abr. 2008.

TURRENT, F. A. Uso de una matriz mixta para la optimización de cinco a ocho factores controlables de la producción, Chapingo: Rama de Suelos, Colégio de Postgraduados, 1979. 65 p. (Boletim técnico, 6).

VENTURIN, N. et al. Adubação mineral do Angico-Amarelo (Peltophorum dubium (SPRENG.) TAUB.). Pesquisa Agropecuária Brasileira, Brasília, v. 34, n. 3, p. 441-448, mar. 1999. 